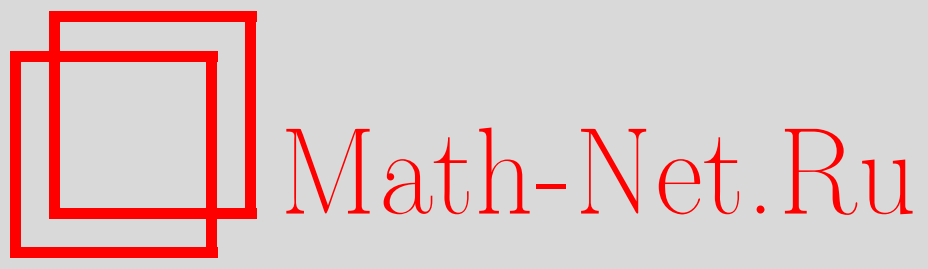

В. А. Менегатту, Ж. К. Феррейра, Гильбертовы пространства, порожденные воспроизводящими ядрами, заданными на топологических пространствах, Функи. анализ и его прил., 2012, том 46, выпуск 2, 89-91

DOI: https://doi.org/10.4213/faa3074

Использование Общероссийского математического портала MathNet.Ru подразумевает, что вы прочитали и согласны с пользовательским соглашением http://www . mathnet.ru/rus/agreement

Параметры загрузки:

IP : 3.81 .55 .215

26 апреля 2023 г., 07:16:10

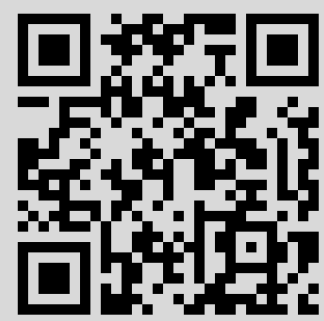


УДК 917.98

\title{
Гильбертовы пространства, порожденные воспроизводящими ядрами, заданными на топологических пространствах*
}

\author{
(C) 2012. В. А. МенеГАтту, Ж. К. ФЕРРейРА
}

1. Основные определения. Пусть $X$ - непустое множество и $K: X \times$ $X \rightarrow \mathbb{C}$ - положительно определенное ядро на $X$; это означает, что матрица $\left(K\left(x_{i}, x_{j}\right)\right)_{n \times n}$ является неотрицательно определенной для всех $n \geqslant 1$ и всех наборов $x_{1}, \ldots, x_{n} \in X$.

Гильбертово пространство $\mathscr{H}_{K}$, порожденное таким ядром $K$, - это (по определению) наименьшее гильбертово пространство, содержащее множество $\left\{K^{x}:=K(\cdot, x): x \in X\right\}$ со скалярным произведением $\left\langle K^{x}, K^{y}\right\rangle_{K}:=K(y, x)$. Это пространство характеризуется следующим воспроизводящим свойством: $f(x)=\left\langle f, K^{x}\right\rangle_{K}, f \in \mathscr{H}_{K}, x \in X$. Для случая, когда на $X$ задана мера $\nu$ и интегральный оператор $\mathscr{K}: L^{2}(X, \nu) \rightarrow L^{2}(X, \nu)$, заданный формулой $\mathscr{K}(f)(x)=$ $\langle K(x, \cdot), f\rangle_{2}, x \in X$, корректно определен, имеются классические результаты относительно $K, \mathscr{K}, \mathscr{H}_{K}$; см. [2], [4], [5].

В этой работе предполагается, что $X$ - топологическое пространство, удовлетворяющее первой аксиоме счетности либо локально компактное. На нем вводится такая (полная) борелевская мера $\nu$, что каждое непустое открытое множество из $X$ имеет положительную меру и каждая точка $x \in X$ принадлежит открытому множеству конечной меры.

Мы будем говорить, что ядро $K$ является $L^{2}(X, \nu)$-положительно определенным, если указанный выше оператор $\mathscr{K}$ корректно определен, ограничен и удовлетворяет неравенству $\langle\mathscr{K}(f), f\rangle_{2} \geqslant 0, f \in L^{2}(X, \nu)$. Заметим, что непрерывное $L^{2}(X, \nu)$-положительно определенное ядро есть положительно определенное ядро в обычном смысле [3]. Введем обозначение $\kappa(x)=K(x, x), x \in X$. Справедливо неравенство $|f(x)|=\left|\left\langle f, K^{x}\right\rangle_{K}\right| \leqslant \kappa(x)^{1 / 2}\|f\|_{K}, f \in \mathscr{H}_{K}, x \in X$.

Теорема 1.1. Предположим, что каждая из функиий $K^{y}$ непрерывна.

(i) Если последовательность $\left\{f_{n}\right\}$ сходится $\kappa f$ в пространстве $\mathscr{H}_{K}$, то она сходится равномерно на компактных множествах.

(ii) $\mathscr{H}_{K} \subset C(X)$.

Доказательство. Заметим, что

$$
\left|f_{n}(x)-f(x)\right|=\left|\left\langle f_{n}-f, K^{x}\right\rangle_{K}\right| \leqslant\left\|f_{n}-f\right\|_{K} \kappa(x)^{1 / 2}, \quad x \in X,
$$

и если последовательность функций $f_{n}=\sum_{i=1}^{j_{n}} c_{i} K^{x_{i}}, c_{i} \in \mathbb{C}, x_{i} \in X$, сходится к $f$ в $\mathscr{H}_{K}$, то она сходится равномерно на компактных множествах. Теперь требуемый результат вытекает из условий теоремы.

Всюду в дальнейшем мы будем считать, что $K$ есть непрерывное $L^{2}(X, \nu)$-положительно определенное ядро. Если при этом функция $\kappa$ ограничена, то отображение включения $i: \mathscr{H}_{K} \hookrightarrow C(X)$ тоже ограничено.

*Исследования первого автора, V. A. Menegatto, были поддержаны FAPESP, грант 2010.19734-6, исследования второго автора, J. C. Ferreira, - FAPEMIG, грант APQ-03911-10. 
Теорема 1.2. Если $\kappa \in L^{1}(X, \nu)$, mо

(i) $\mathscr{H}_{K} \subset C(X) \cap L^{2}(X, \nu) u\|f\|_{2} \leqslant\|\kappa\|_{1}^{1 / 2}\|f\|_{K}, f \in \mathscr{H}_{K}$;

(ii) $\mathscr{K}(f) \in \mathscr{H}_{K} u\langle\mathscr{K}(f), g\rangle_{K}=\langle f, g\rangle_{2}, g \in \mathscr{H}_{K}, f \in L^{2}(X, \nu)$.

Доказательство. Если $\psi_{f}(g)=\langle g, f\rangle_{2}, g \in \mathscr{H}_{K}, f \in L^{2}(X, \nu)$, то $\left|\psi_{f}(g)\right| \leqslant$ $\|f\|_{2}\|\kappa\|_{1}^{1 / 2}\|g\|_{K}$. Так как существует такой вектор $h \in \mathscr{H}_{K}$, что $\psi_{f}(g)=\langle g, h\rangle_{K}$, $g \in \mathscr{H}_{K}$, то $h(x)=\left\langle h, K^{x}\right\rangle_{K}=\left\langle f, K^{x}\right\rangle_{2}=\mathscr{K}(f)(x), x \in X$.

2. Теория Мерсера. Если вектор-функция $x \in X \rightarrow K(x, \cdot) \in L^{2}(X, \nu)$ принадлежит пространству $C\left(X, L^{2}(X, \nu)\right)$, то из определения оператора $\mathscr{K}$ следует, что его область значений содержится в пространстве $C(X)$. Если $\mathscr{K}$ компактен, то он самосопряжен и имеет спектральное разложение $\mathscr{K}(f)=$ $\sum_{n=1}^{\infty} \lambda_{n}\left\langle f, \phi_{n}\right\rangle_{2} \phi_{n}, f \in L^{2}(X, \nu)$, где набор $\left\{\phi_{n}\right\}$ ортонормирован в $L^{2}(X, \nu)$, последовательность $\left\{\lambda_{n}\right\}$, убывая, стремится к нулю, $\left\{\lambda_{n} \phi_{n}\right\} \in C(X)$ и при этом справедливо равенство

$$
K(x, y)=\sum_{n=1}^{\infty} \lambda_{n} \phi_{n}(x) \overline{\phi_{n}(y)}, \quad x, y \in X .
$$

Такого типа ядро $K$ назовем ядром Мерсера. Проверка того, что непрерывное ядро является ядром Мерсера, оказывается непростой задачей - как в ситуации, рассматриваемой в настоящей работе, так и в случае, рассмотренном в [4].

Теорема 2.1 (теорема Мерсера I). Ecли $\kappa \in L^{1}(X, \nu)$, mo K есть ядро Мерсера.

Доказательство. Если ядро $K$ квадратично интегрируемо, то оператор $\mathscr{K}=\mathscr{K}^{*}$ компактен. В этом случае, согласно теореме 1.2 , область значений оператора $\mathscr{K}$ лежит в $C(X)$. Остается применить аргументы из доказательства теоремы 2.4 из [3], приспособив их к тем условиям, которые мы наложили на пространство $X$.

В следующих двух теоремах мы предполагаем, что функция $\kappa$ принадлежит пространству $L^{1}(X, \nu)$.

Теорема 2.2. Множество $\left\{\lambda_{n}^{1 / 2} \phi_{n}: \lambda_{n} \neq 0\right\}$ есть ортонормированный базис в $\mathscr{H}_{K}$.

Доказательство. Согласно теореме 1.2, $\left\langle\lambda_{n} \phi_{n}, \phi_{m}\right\rangle_{K}=\left\langle\mathscr{K}\left(\phi_{n}\right), \phi_{m}\right\rangle_{K}=$ $\left\langle\phi_{n}, \phi_{m}\right\rangle_{2}=\delta_{m, n}$ при $\lambda_{n} \neq 0$. Следовательно, набор $\left\{\lambda_{n}^{1 / 2} \phi_{n}: \lambda_{n} \neq 0\right\}$ ортонормирован в $\mathscr{H}_{K}$. Если вектор $f \in \mathscr{H}_{K}$ удовлетворяет условию $\left\langle f, \phi_{n}\right\rangle_{K}=0$, $n=1,2, \ldots$, то из соотношения

$$
K^{x}=\sum_{n=1}^{\infty} \lambda_{n} \overline{\phi_{n}(x)} \phi_{n}=\sum_{n=1}^{\infty}\left\langle K^{x}, \phi_{n}\right\rangle_{K} \lambda_{n} \phi_{n}, \quad x \in X,
$$

вытекает, что

$f(x)=\left\langle f, K^{x}\right\rangle_{K}=\left\langle f, \sum_{n=1}^{\infty}\left\langle K^{x}, \phi_{n}\right\rangle_{K} \lambda_{n} \phi_{n}\right\rangle_{K}=\sum_{n=1}^{\infty}\left\langle f, \phi_{n}\right\rangle_{K} \lambda_{n} \phi_{n}(x)=0, \quad x \in X$.

Таким образом, множество $\left\{\lambda_{n}^{1 / 2} \phi_{n}: \lambda_{n} \neq 0\right\}$ есть ортонормированный базис в $\mathscr{H}_{K}$ 
Для дальнейшего введем оператор $\mathscr{K}^{1 / 2}: L^{2}(X, \nu) \rightarrow C(X) \subset L^{2}(X, \nu)$ формулой $\mathscr{K}^{1 / 2}\left(\phi_{n}\right)=\lambda_{n}^{1 / 2} \phi_{n}, n=1,2, \ldots$ (см. [3]). Для любого множества $B \subset$ $L^{2}(X, \nu)$ обозначим через $\bar{B}$ его замыкание и через $B^{\perp}$ ортогональное дополнение.

Теорема 2.3. Оператор $\mathscr{K}^{1 / 2}$ осуществляет изометрический изоморфизм замыкания линейной оболочки множества $\left\{K^{x}: x \in X\right\}$ в $L^{2}(X, \nu)$ на $\mathscr{H}_{K}$.

Доказательство. Положим $\mathscr{D}_{K}:=\operatorname{span}\left\{K^{x}: x \in X\right\}$ и $V_{K}=\operatorname{span}\left\{\lambda_{n} \phi_{n}\right\}$. Так как $K$ есть ядро Мерсера, то $\mathscr{D}_{K} \subset \overline{V_{K}}$ и ${\overline{V_{K}}}^{\perp} \subset \overline{\mathscr{D}_{K}} \stackrel{\perp}{ }$. Если $f \in \mathscr{D}_{K}^{\perp}$, то $\mathscr{K}(f)(x)=\left\langle f, K^{x}\right\rangle_{2}=0, x \in X$, т. е. $\mathscr{K}(f)=0$. Из теоремы 1.2 вытекает, что $\left\langle f, \phi_{n}\right\rangle_{2}=\left\langle\mathscr{K}(f), \phi_{n}\right\rangle_{K}=0, n=1,2, \ldots$, и $\langle f, g\rangle_{2}=0, g \in \overline{V_{K}}$. Следовательно, ${\overline{V_{K}}}^{\perp} \supset \overline{\mathscr{D}}_{K} \perp$. Заметим теперь, что если $g \in \mathscr{H}_{K}$, то $g=\sum_{n=1}^{\infty} \beta_{n} \lambda_{n}^{1 / 2} \phi_{n}=$ $\mathscr{K}^{1 / 2}(f)$, где $f=\sum_{n=1}^{\infty} \beta_{n} \phi_{n} \in \overline{D_{K}}$; осталось применить равенство Парсеваля.

В завершение этой заметки заменим условие интегрируемости функции $\kappa$ условием плотности множества $L_{c}^{2}(X)$ (состоящего из функций с компактным носителем) в $L^{2}(X, \nu)$. Условие плотности выполняется, если $\nu$ - радонова меpa, а также если $X$ - специального вида метрическое пространство (см. [4]).

Теорема 2.4. Если $\left\{f_{n}\right\}$ - последовательность из $L_{c}^{2}(X)$, сходящаяся $\kappa$ элементу $f$ в $L^{2}(X, \nu)$, то $\mathscr{K}(f)$ - непрерывная функиия, лежащая в $\mathscr{H}_{K}, u$ $\langle\mathscr{K}(f), g\rangle_{K}=\lim _{n \rightarrow \infty}\left\langle f_{n}, g\right\rangle_{2} \partial \Omega я g \in \mathscr{H}_{K}$.

Доказательство. Повторяя доказательство теоремы 1.2 , получаем, что $\mathscr{K}\left(f_{n}\right) \in \mathscr{H}_{K}$ и $\left\langle\mathscr{K}\left(f_{n}\right), g\right\rangle_{K}=\left\langle f_{n}, g\right\rangle_{2}$. Следовательно, $\left\|\mathscr{K}\left(f_{n}\right)-\mathscr{K}\left(f_{m}\right)\right\|_{K}^{2} \leqslant$ $\|\mathscr{K}\|_{2}\left\|f_{n}-f_{m}\right\|_{2}^{2}$, откуда вытекает, что последовательность $\left\{\mathscr{K}\left(f_{n}\right)\right\}$ сходится в $\mathscr{H}_{K}$.

Предыдущие результаты и доказательство теоремы 2.1 приводят к следующему результату.

Теорема 2.5. Если оператор $\mathscr{K}$ компактен, то K есть ядро Мерсера.

Так как в случае квадратично интегрируемого ядра $K$ оператор $\mathscr{K}$ компактен, то теорема 2.5 показывает, что ограничения, наложенные в [1], [4], являются излишними либо чрезмерно жесткими. В заключение заметим, что в случае, когда $K$ - ядро Мерсера, можно доказать аналоги теорем 1.2. и 2.3.

\section{ЛиТЕРАТУРА}

[1] J. Buescu, J. Math. Anal. Appl., 296:1 (2004), 244-255. [2] F. Cucker, S. Smale, Bull. Amer. Math. Soc. (N.S.), 39:1 (2002), 1-49. [3] J. C. Ferreira, V. A. Menegatto, Integral Equation Operator Theory, 64:1 (2009), 61-81. [4] Hongwei Sun, J. Complexity, 21:3 (2005), 337-349. [5] Hongwei Sun, Qiang Wu, Math. Comput. Modelling, 49:1-2 (2009), 276-285.

Departamento de Matemática,

Instituto de Ciências Matemáticas e de Computação,

Universidade de São Paulo

e-mail: menegatt@icmc.usp.br

Instituto de Ciências Exatas, Universidade Federal de Alfenas e-mail: jose.ferreira@unifal-mg.edu.br
Поступило в редакцию 15 октября 2010 г. 